\title{
The Physiologic Effects of an Acute Bout of Supramaximal High-Intensity Interval Training Compared with a Continuous Exercise Bout in Patients with COPD
}

\author{
Scotty J. Butcher, ${ }^{1}$ Madison T. Yurach, ${ }^{2}$ Nichole M. Heynen, ${ }^{1}$ Brendan J. Pikaluk, ${ }^{2}$ \\ Karla J. Horvey, ${ }^{1}$ Ron Clemens, ${ }^{3}$ and Darcy D. Marciniuk ${ }^{3}$ \\ ${ }^{1}$ School of Physical Therapy, University of Saskatchewan, Saskatoon, SK, Canada S7N 0W3 \\ ${ }^{2}$ College of Kinesiology, University of Saskatchewan, Saskatoon, SK, Canada S7N 5B2 \\ ${ }^{3}$ Division of Respirology, Critical Care, and Sleep Medicine, University of Saskatchewan, Saskatoon, SK, Canada S7N 0W8 \\ Correspondence should be addressed to Scotty J. Butcher; scotty.butcher@usask.ca
}

Received 25 May 2013; Revised 23 August 2013; Accepted 10 September 2013

Academic Editor: Rio Dumitrascu

Copyright (C) 2013 Scotty J. Butcher et al. This is an open access article distributed under the Creative Commons Attribution License, which permits unrestricted use, distribution, and reproduction in any medium, provided the original work is properly cited.

\begin{abstract}
This study compared physiological responses and work performed during a supramaximal high-intensity interval exercise training session (HIIT) and a constant work rate (CWR) exercise session. Fourteen patients with COPD (mean FEV $53 \pm 13 \%$ predicted $( \pm$ SD) $)$ completed an incremental cardiopulmonary exercise test (CPET) and a steep ramp anaerobic test (SRAT) and then two exercise bouts to symptom limitation on separate days, in random order: (1) a CWR trial at $80 \%$ of CPET peak work rate (mean $63 \pm 15 \mathrm{~W}$ ) and (2) a HIIT trial using repeats of $30 \mathrm{~s}$ at $70 \%$ of SRAT peak work rate (mean $112 \pm 29 \mathrm{~W}$ ) followed by $90 \mathrm{~s}$ at $20 \%$ of CPET peak work rate. Subjects ceased exercise primarily due to dyspnea for both HIIT and CWR (64\% vs. 57\%, resp.). End-exercise $V_{E}$, HR, dyspnea, and leg fatigue were similar between the two exercise protocols. Average work rate was lower in HIIT than CWR ( 32 vs. $63 \mathrm{~W}, P<0.05)$; however, subjects performed HIIT longer $(542$ vs. $202 \mathrm{~s}, P<0.05)$ and for greater total work (23.3 vs. $12.0 \mathrm{~kJ}, P<0.05)$. The supramaximal HIIT protocol was well tolerated and demonstrated similar maximal physiologic responses to constant work rate exercise, but with greater leg muscle work performed and greater peak exercise intensity.
\end{abstract}

\section{Introduction}

Chronic obstructive pulmonary disease (COPD) is recognized as one of the fastest growing chronic diseases leading to mortality [1]. The chief symptom of COPD is dyspnea on exertion leading to exercise intolerance and deconditioning [2]. Exercise training is a vital component of rehabilitation for patients with COPD; however, limitations on ventilation during exercise often prevent patients from attaining maximal cardiovascular and/or muscular responses. These limitations lead to significant deconditioning [3] and can impair their ability to physiologically adapt to training.

High-intensity interval training (HIIT) is a method of exercise training whereby short periods of high intensity work are interspersed with periods of lower intensity recovery or rest. In many clinical populations, HIIT has achieved greater physiological adaptations than traditional exercise rehabilitation utilizing continuous constant work rate (CWR) exercise [4-7]. Although HIIT has been proposed as a method of addressing the reduced training effects in patients with COPD $[8,9]$, studies reported to date demonstrate that the physiological adaptations with HIIT are no different than those with CWR training [9-11], although HIIT may be better tolerated by patients than CWR training [11]. These studies, however, have typically set work rate for the high-intensity work interval using peak power output on a cardiopulmonary exercise test (CPET) or peak rate of oxygen consumption $\left(\mathrm{VO}_{2}\right.$ peak) test. Due to the ventilatory limitations observed during exercise in patients with COPD, however, CPET work rates typically underestimate their metabolic, muscular, and cardiovascular capacity $[8,12,13]$ and may be less effective for the prescription of optimal HIIT work phase intensity.

A potential solution to this problem would be to assess work phase intensity using the results of an anaerobic test 
such as the steep ramp anaerobic test (SRAT) [14]. This reliable [13] test increases work rate much more quickly than CPET and has been demonstrated to reflect anaerobic abilities better than CPET in patients with COPD $[12,13]$. This test was developed for the use of HIIT prescription in patients with heart failure [14] and has been utilized in a similar manner in patients with COPD [10]. In a study examining the responses to HIIT in patients with COPD, Puhan and colleagues [10] utilized $50 \%$ of the peak work rate achieved on the SRAT to set work phase intensity. This intensity corresponded to approximately $100 \%$ of CPET peak work rate, which was similar to previous studies of HIIT in patients with COPD. Not surprisingly, then, they found that HIIT did not result in greater physiological increases compared with CWR training [10]. In healthy individuals, HIIT protocols that include a high intensity interval of up to $100 \%$ of CPET peak work rate are effective in improving aerobic performance. In order to achieve anaerobic gains, however, the high intensity interval likely needs to be above CPET peak work rate [15]. Based on our work indicating that high intensity, anaerobic functional performance is better reflective by anaerobic tests in patients with COPD [12], we propose that work phase intensities higher than peak power obtained on a CPET are needed to elicit greater physiological adaptations to HIIT than those observed by continuous training. We further postulate that these higher work rates delivered in short intervals will not place greater physiologic stress on the respiratory system. To our knowledge, this type of protocol design has not been examined in patients with COPD.

The purpose of the present study was to compare the acute physiological responses during a novel SRAT-based, supramaximal high-intensity interval exercise training (HIIT) session with those during a continuous CWR exercise training session in patients with COPD. We hypothesized that this HIIT protocol would be as tolerable as CWR exercise and would result in a greater amount of work performed with similar physiological responses.

\section{Materials and Methods}

2.1. Participants. Sixteen participants ( 9 female) with moderate to severe COPD, as defined by the Canadian Thoracic Society Guidelines [1], were recruited from the local Pulmonary Rehabilitation Program. Ethical approval was granted by the appropriate institutional ethics review boards. Subjects were excluded if there was evidence of resting hypoxemia $\left(\mathrm{PaO}_{2}<60 \mathrm{mmHg}\right.$ if available or $\left.\mathrm{SaO}_{2}<88 \%\right)$, they required continuous supplemental oxygen usage, their oxygen desaturation decreased to less than $80 \%$ during exercise, they exhibited any contraindications to exercise testing [16], or if there was a history of known or suspected cardiac disease or musculoskeletal concerns that might limit the ability of these participants to perform active exercise. Two recruited participants were excluded; therefore, 14 participants (8 female) completed the study. Preliminary sample size calculations indicated that 8 participants would be required to observe differences between CWR and HIIT protocols. Participant characteristics are presented in Table 1.
TABle 1: Participant demographics, pulmonary function, and responses to the CPET and SRAT.

\begin{tabular}{|c|c|c|}
\hline Gender (M/F, $n)$ & $6 / 8$ & \\
\hline Age (yr) & $68.6 \pm 11.9$ & $(49-78)$ \\
\hline Weight $(\mathrm{kg})$ & $80.7 \pm 15.0$ & $(57-96)$ \\
\hline Body mass index $\left(\mathrm{kg} \cdot \mathrm{m}^{-2}\right)$ & $29.6 \pm 3.6$ & $(23-33)$ \\
\hline $\mathrm{FEV}_{1}(\mathrm{~L})$ & $1.26 \pm 0.31$ & $(0.80-1.65)$ \\
\hline $\mathrm{FEV}_{1}(\%$ predicted $)$ & $52.6 \pm 12.7$ & $(46-75)$ \\
\hline $\mathrm{FEV}_{1} / \mathrm{FVC}(\%)$ & $59.2 \pm 11.8$ & $(38-54)$ \\
\hline TLC (L) & $6.44 \pm 1.48$ & $(4.33-7.99)$ \\
\hline TLC (\% predicted) & $118 \pm 17$ & $(98-142)$ \\
\hline $\mathrm{RV}(\mathrm{L})$ & $1.72 \pm 0.91$ & $(1.02-3.24)$ \\
\hline RV (\% predicted) & $142 \pm 38$ & $(105-177)$ \\
\hline $\mathrm{D}_{\mathrm{L}} \mathrm{CO}(\%$ predicted $)$ & $67.8 \pm 14.1$ & $(51-87)$ \\
\hline CPET $V \mathrm{O}_{2}$ peak $\left(\mathrm{L} \cdot \mathrm{min}^{-1}\right)$ & $1.14 \pm 0.41$ & $(0.66-1.56)$ \\
\hline CPET $V \mathrm{O}_{2}$ peak $\left(\mathrm{mL} \cdot \mathrm{kg}^{-1} \cdot \mathrm{min}^{-1}\right)$ & $14.1 \pm 3.18$ & $(8.2-18.6)$ \\
\hline CPET WRpeak (W) & $78.8 \pm 31.2$ & $(37-116)$ \\
\hline SRAT $V \mathrm{O}_{2}$ peak $\left(\mathrm{L} \cdot \mathrm{min}^{-1}\right)$ & $1.11 \pm 0.44$ & $(0.57-1.54)$ \\
\hline SRAT $V \mathrm{O}_{2}$ peak $\left(\mathrm{mL} \cdot \mathrm{kg}^{-1} \cdot \mathrm{min}^{-1}\right)$ & $13.2 \pm 0.56$ & $(7.6-18.3)$ \\
\hline SRAT WRpeak (W) & $161.2 \pm 42.7$ & $(100-195)$ \\
\hline
\end{tabular}

Mean \pm SD (range).

2.2. Design. This study employed a randomized, cross-over design to assess the acute physiological responses and performance during HIIT and CWR exercise. Participants attended four sessions to the laboratory, each at least 48 hours apart. In session 1, a medical history and measures of height, weight, resting blood pressure, heart rate, and oxygen saturation were taken. As well, participants completed pulmonary function testing and a CPET (as described below). In session 2, participants completed the SRAT. In sessions 3 and 4 , participants performed one of the exercise protocols in each visit, completed in a random order.

2.3. Pulmonary Function Testing. Spirometry $\left(\mathrm{FEV}_{1}, \mathrm{FVC}\right)$, lung volumes (TLC), and diffusion capacity (DLCO) were measured to ATS/ERS standards [17-19] using a Vmax AutoBox (SensorMedics, Yorba Linda, CA). Maximal voluntary ventilation $(\mathrm{MVV})$ was estimated by $\mathrm{FEV}_{1} \times 35$ [16].

2.4. Cardiopulmonary Exercise Test (CPET). A maximal cycle ergometry test was performed to patient exhaustion using the standards of the American Thoracic Society-American College of Chest Physicians [16]. The test began with a one minute warm-up of unloaded cycling on an electronicallybraked cycle ergometer (839E, Monark Exercise AB, Vansbro, Sweden). The warm up was followed by ramped incremental increases in work rate until symptom limitation. Based on clinical history and presentation, a work rate increment of 5-15 watts per minute was selected for each participant. Respiratory gases were measured with a metabolic measurement cart with both inspiratory and expiratory flow pneumotachometers (TrueOne, ParvoMedics, Salt Lake City, UT). Oxygen saturation was recorded using pulse oximetry. Peak exercise symptoms of dyspnea and leg fatigue were recorded using a modified Borg scale for perceived exertion [20]. Peak rate of oxygen consumption $\left(\mathrm{VO}_{2}\right.$ peak $)$ and peak work rate (WR) were recorded. 
2.5. Steep Ramp Anaerobic Test (SRAT). The SRAT was performed as described previously $[12,13]$. Briefly, the test began with a two minute unloaded warm up followed by ramped increases of 25 watts every 10 seconds until volitional fatigue. As for the CPET, $V \mathrm{O}_{2}$ peak and peak WR were recorded for the SRAT.

2.6. HIIT and CWR Exercise Sessions. For each of the exercise sessions, participants were instructed to exercise as long as possible, until symptom limitation or volitional exhaustion. The time to exhaustion or symptom limitation (TTE/SL), symptoms of dyspnea and leg fatigue, total work performed, and reason for exercise cessation (either dyspnea, leg fatigue, or both) were recorded or calculated. Each session was preceded by a 3 minute unloaded warm up on the cycle ergometer. During each test, expiratory gases were recorded as per the CPET. $V \mathrm{O}_{2}$ peak, peak WR, peak ventilation $\left(V_{E}\right)$, peak heart rate (HR), and end-exercise inspiratory capacity (IC), were recorded. In addition, oxygen saturation, heart rate, and IC were recorded every 2 minutes and at exercise cessation. The constant work rate (CWR) protocol was performed at $80 \%$ of peak WR obtained on the CPET. The highintensity interval training (HIIT) protocol was performed for 30 seconds at $70 \%$ of SRAT peak WR alternating with 90 seconds at $20 \%$ of CPET peak WR. Based on previous work $[10,12,13]$, it was expected that SRAT peak WR would be approximately $200 \%$ of the CPET peak WR. The work interval in the HIIT protocol would therefore correspond to approximately $140 \%$ of peak WR on the CPET but would be based upon each participant's anaerobic capacity [13]. This protocol has been chosen based on the work of Meyer et al. [14] who determined the work interval for HIIT on the results of a SRAT and the recovery interval on the results of a CPET. Although this approach requires two separate tests, it allows for the high intensity interval to be more appropriately based on anaerobic abilities, whereas the recovery interval would be based on aerobic abilities [14].

2.7. Statistical Analysis. Peak exercise data, as well as total work performed, were described as means and standard deviations. In addition, for each participant, the point of equal work performed (isowork) between the two protocols was determined, and the exercise data that corresponded with this time point were described similarly. For the TTE/SL and total work performed in each protocol, a paired Student's $t$-test was performed to compare protocol means. For all other exercise data, a repeated measures ANOVA was used to compare the exercise data at the exercise cessation and at the time of isowork. Tukey's post hoc analysis was used when significant main effects were observed. Statistical significance was set at $\alpha=0.05$.

\section{Results}

3.1. Exercise Performance Characteristics. The SRAT resulted in peak work rates of $204 \%$ of the CPET peak work rates; therefore, the HIIT work interval was performed at $112 \mathrm{~W}$ (142\% of CPET peak work rate) (Tables 1 and 2). The CWR
TABLE 2: Performance data on the HIIT and CWR protocols.

\begin{tabular}{lcc}
\hline & HIIT & CWR \\
\hline Work interval (W) & $112 \pm 29$ & $63 \pm 15^{*}$ \\
Recovery interval (W) & $16 \pm 5$ & N/A \\
Average WR (W) & $32 \pm 8$ & $63 \pm 15^{*}$ \\
TTE/SL (s) & $542 \pm 293$ & $202 \pm 82^{*}$ \\
Total work performed (kJ) & $23.3 \pm 9.5$ & $12.0 \pm 6.0^{*}$ \\
\hline
\end{tabular}

Mean \pm SD. ${ }^{*} P<0.05$ versus HIIT. WR: work rate; TTE/SL: time to exhaustion or symptom limitation; HIIT: high-intensity interval training session; CWR: constant work rate session.

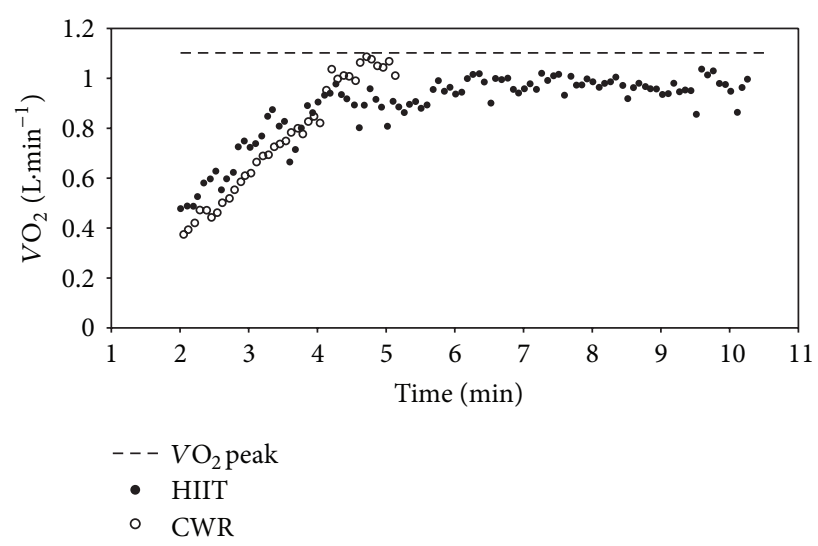

FIGURE 1: Oxygen consumption responses for a representative participant. Each data point represents 5 second averaging.

protocol work rate was much lower than the peak work rate of the HIIT protocol; however, it resulted in a higher overall average work rate than HIIT. Participants completed $170 \%$ more time and 95\% more total work with the HIIT protocol.

3.2. Physiological and Perceptual Data. There were no differences in the peak $V \mathrm{O}_{2}, V_{E}$, IC, $S_{P} \mathrm{O}_{2}$, dyspnea, or exertion values (Table 3). Both exercise protocols resulted in peak $\mathrm{VO}_{2}$ values greater than $90 \%$ of $V \mathrm{O}_{2} \max$ and peak $V_{E}$ values greater than $85 \%$ of predicted MVV. Both $V \mathrm{O}_{2}$ and $V_{E}$ during the HIIT protocol, however, plateaued early during the session and were sustained for the duration of exercise (a representative example is shown in Figures 1 and 2).

All participants exercised longer and completed more work using the HIIT protocol than the CWR protocol; therefore, all isowork data were recorded from values taken during the HIIT protocol. At the point of isowork, participants had a lower ventilation rate, a higher inspiratory capacity, and lower dyspnea score than end-exercise values on both protocols, indicating lower levels of ventilatory limitation. Participants described dyspnea as the primary reason for exercise cessation for both protocols (Table 3).

\section{Discussion}

The primary objectives of this study were to examine the physiological responses to a novel, supramaximal highintensity interval training protocol in patients with COPD 
TABLE 3: Exercise physiologic and perceptual data.

\begin{tabular}{lccc}
\hline & HIIT-end & HIIT-isowork & CWR \\
\hline $\mathrm{HR}(\mathrm{bpm})$ & $121 \pm 31$ & $101 \pm 36$ & $120 \pm 29$ \\
$V_{E}\left(\mathrm{~L} \cdot \mathrm{min}^{-1}\right)$ & $39 \pm 11$ & $32 \pm 15^{*}$ & $41 \pm 13$ \\
$\mathrm{VO}_{2}\left(\mathrm{~L} \cdot \mathrm{min}^{-1}\right)$ & $1.03 \pm 0.26$ & $0.97 \pm 0.36$ & $1.05 \pm 0.41$ \\
$\mathrm{IC}(\% \mathrm{TLC})$ & $25 \pm 6$ & $38 \pm 6^{*}$ & $27 \pm 8$ \\
$S_{P} \mathrm{O}_{2}(\%)$ & $92 \pm 3$ & $94 \pm 2$ & $92 \pm 2$ \\
Dyspnea & $5.7 \pm 1.8$ & $4.9 \pm 1.6$ & $5.8 \pm 1.4$ \\
Exertion & $4.2 \pm 1.6$ & $3.4 \pm 1.3^{*}$ & $4.3 \pm 0.4$ \\
& Dyspnea 9 & & Dyspnea 8 \\
Reason for cessation $(n)$ & Leg fatigue 3 & Noth 2 & Leg fatigue 3 \\
& Both 3 & B \\
\hline
\end{tabular}

${ }^{*} P<0.05$ versus HIIT-end. HR: heart rate; $V_{E}$ : ventilation; $V \mathrm{O}_{2}$ : rate of oxygen consumption; IC: inspiratory capacity; $S_{P} \mathrm{O}_{2}$ : oxygen saturation; HIIT: highintensity interval training session; CWR: constant work rate session.

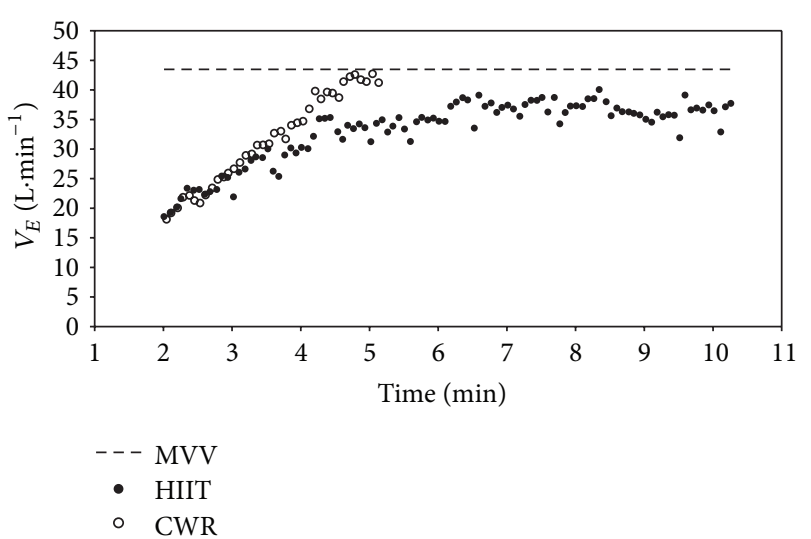

FIGURE 2: Ventilation responses for a representative participant. Each data point represents 5 second averaging.

and to compare this protocol with a high-intensity, continuous aerobic exercise protocol. Our results demonstrated that our protocol of HIIT above CPET peak work rate was tolerable and allowed patients to complete almost double the total work of $80 \%$ CWR exercise prior to symptom limitation. This greater peak work rate and total work performed, however, resulted in similar end-exercise physiological and symptomatic responses.

It is important to note that the results of this study are applicable only to the specific protocols utilized; different intensities and work to rest ratios would likely result in different results. For example, these results support some of the findings demonstrated by Puhan et al. [10] who examined the long term training effect of HIIT at $50 \%$ of SRAT peak power compared with CWR training at 70\% of CPET peak power. These authors reported the work performance of both protocols and found that the average training work was $25.6 \mathrm{~kJ}$ for the HIIT protocol and $33.5 \mathrm{~kJ}$ for the CWR protocol [10] (versus 23.3 and $12.0 \mathrm{~kJ}$, resp., in our study). These two HIIT protocols elicited similar total work, despite the protocol used by Puhan being conducted at a lower intensity work interval $(\sim 100 \%$ CPET) and lower overall average work rate. Thus, using our supramaximal protocol, patients were able to achieve a similar amount of total work with the physiologic benefit of a higher work interval intensity. It is likely, then, that our protocol may result in greater physiological adaptation. This suggestion requires validation utilizing our training protocol in a longitudinal study of training effectiveness.

In regard to the constant work rate protocols, not surprisingly, our high-intensity protocol ( $80 \%$ of CPET peak work rate) resulted in much less work performed than the moderate intensity (70\% of CPET) used by Puhan et al. [10]. This finding suggests that lower intensities of continuous exercise result in greater amounts of work tolerated by patients and may be superior to higher intensities of continuous exercise for increasing exercise duration. If increases in intensity are desired with training, however, the HIIT protocol utilized in this study is likely a more appropriate option for clinicians in pulmonary rehabilitation. The optimal combination of work intensity, work to rest ratios, and session duration for specific adaptations has yet to be determined and should be the focus of future research.

HIIT has been shown to be superior to CWR training for inducing physiological adaptations in many other chronic diseases such as heart failure [5], coronary artery disease $[4,6]$, and metabolic disease [7] and has been suggested to be at least as safe as CWR training [21, 22]. In these populations, even work intensities at $100 \%$ of CPET peak power (or $\mathrm{VO}_{2} \max$ ) result in greater aerobic adaptations. Although not a question is directly answered by the results of this study, we believe that our findings support the suggestion that setting exercise training intensities around peak work rate on a CPET in patients with COPD who are ventilatory limited results in an underestimation of training intensity and will likely not result in high-intensity, anaerobic adaptations that are important for functional performance $[8,12]$.

It is well known that patients typically exhibit submaximal cardiovascular and metabolic responses during symptom limited CPET [16] and that CPET underestimates their muscular capabilities $[12,13]$. Expiratory flow limitation and dynamic hyperinflation constrain the increase in tidal volume and, subsequently, ventilation during exercise [23] and this constraint results in an impairment of the ability to train 
the peripheral muscles [3]. This suggestion is supported by the fact that patients with COPD can typically sustain higher percentages of peak CPET work rate compared with their healthy counterparts, indicating that CPET peak work rate is abnormally blunted [24].

Delaying ventilatory limitation through the use of helium or oxygen breathing allows for greater amounts of work to be performed $[3,25,26]$; however, it may not be a practical strategy for most patients attending rehabilitation. The results of our study, and those of others [27], suggest that interval exercise results in delayed ventilatory limitation for a given amount of work performed and therefore has the potential to result in greater performance. Reports of HIIT in patients with COPD to date have not supported this suggestion [9]; however, it is likely that training intensity is typically lower than that required for optimal adaptation. Using a higher intensity based on anaerobic abilities (as measured by tests such as the SRAT) rather than aerobic abilities (as measured by CPET) may result in greater adaptation than continuous training.

It is important to note that both protocols utilized in this study resulted in an exercise duration that is shorter than is typically recommended for pulmonary rehabilitation training sessions $[8,9]$. In addition, there were no differences between the levels of dyspnea at peak CWR work and at isowork on the HIIT protocol. Because patients will often cease exercise at a given level of dyspnea, it is possible that our HIIT protocol may not be practical in rehabilitation programs. While the long term responses of these protocols are unclear, it is possible that sessions could be divided into a few, shorter intervals grouped into multiple sets with longer recovery periods to increase the session duration. For example, pulmonary rehabilitation professionals could have patients complete $2-4$ high-intensity intervals with our protocol followed by a longer set break (e.g., 5 minutes) and then repeat the 2-4 intervals. The efficacy of this suggestion requires further study.

In conclusion, we found that our novel, supramaximal HIIT protocol was well tolerated by our COPD participants and resulted in greater leg muscle work performed and greater training intensity, with similar physiological responses and ventilatory limitation as compared to highintensity constant work rate exercise. In patients with COPD, it may be possible that basing training intensity on a high percentage of anaerobic capacity and training above CPET peak work rate produces the conditions necessary for greater physiological adaptation with exercise training.

\section{Acknowledgment}

The study was funded by the Saskatchewan Health Research Foundation. All authors report no conflict of interests in this study.

\section{References}

[1] D. E. O’Donnell, S. Aaron, J. Bourbeau et al., "Canadian Thoracic Society recommendations for management of chronic obstructive pulmonary disease-2007 update," Canadian Respiratory Journal, vol. 14, pp. 5B-32B, 2007.

[2] American Thoracic Society and European Respiratory Society, "Skeletal muscle dysfunction in chronic obstructive pulmonary disease: a statement of the American Thoracic Society and European Respiratory Society," American Journal of Respiratory and Critical Care Medicine, vol. 159, supplement 4, pp. S1-S40, 1999.

[3] S. J. Butcher, O. Lagerquist, D. D. Marciniuk, S. R. Petersen, D. F. Collins, and R. L. Jones, "Relationship between ventilatory constraint and muscle fatigue during exercise in COPD," European Respiratory Journal, vol. 33, no. 4, pp. 763-770, 2009.

[4] D. E. R. Warburton, D. C. McKenzie, M. J. Haykowsky et al., "Effectiveness of high-intensity interval training for the rehabilitation of patients with coronary artery disease," American Journal of Cardiology, vol. 95, no. 9, pp. 1080-1084, 2005.

[5] U. Wisløff, A. Støylen, J. P. Loennechen et al., "Superior cardiovascular effect of aerobic interval training versus moderate continuous training in heart failure patients: a randomized study," Circulation, vol. 115, no. 24, pp. 3086-3094, 2007.

[6] O. Rognmo, E. Hetland, J. Helgerud, J. Hoff, and S. A. Slørdahl, "High intensity aerobic interval exercise is superior to moderate intensity exercise for increasing aerobic capacity in patients with coronary artery disease," European Journal of Cardiovascular Prevention and Rehabilitation, vol. 11, no. 3, pp. 216-222, 2004.

[7] A. E. Tjønna, S. J. Lee, Ø. Rognmo et al., "Aerobic interval training versus continuous moderate exercise as a treatment for the metabolic syndrome: a pilot study," Circulation, vol. 118, no. 4, pp. 346-354, 2008.

[8] S. J. Butcher and R. L. Jones, "The impact of exercise training intensity on change in physiological function in patients with chronic obstructive pulmonary disease," Sports Medicine, vol. 36, no. 4, pp. 307-325, 2006.

[9] M. K. Beauchamp, M. Nonoyama, R. S. Goldstein et al., "Interval versus continuous training in individuals with chronic obstructive pulmonary disease- a systematic review," Thorax, vol. 65, no. 2, pp. 157-164, 2010.

[10] M. A. Puhan, G. Büsching, H. J. Schünemann, E. VanOort, C. Zaugg, and M. Frey, "Interval versus continuous highintensity exercise in chronic obstructive pulmonary disease: a randomized trial," Annals of Internal Medicine, vol. 145, no. 11, pp. 816-825, 2006.

[11] I. Vogiatzis, S. Nanas, and C. Roussos, "Interval training as an alternative modality to continuous exercise in patients with COPD," European Respiratory Journal, vol. 20, no. 1, pp. 12-19, 2002.

[12] S. J. Butcher, B. J. Pikaluk, R. L. Chura, M. J. Walkner, J. P. Farthing, and D. D. Marciniuk, "Associations between isokinetic muscle strength, high-level functional performance, and physiological parameters in patients with chronic obstructive pulmonary disease," International Journal of Chronic Obstructive Pulmonary Disease, vol. 7, pp. 537-542, 2012.

[13] R. L. Chura, D. D. Marciniuk, R. Clemens, and S. J. Butcher, "Test-retest reliability and physiological responses associated with the steep ramp anaerobic test in patients with COPD," Pulmonary Medicine, vol. 2012, Article ID 653831, 6 pages, 2012.

[14] K. Meyer, L. Samek, M. Schwaibold et al., "Interval training in patients with severe chronic heart failure: analysis and recommendations for exercise procedures," Medicine and Science in Sports and Exercise, vol. 29, no. 3, pp. 306-312, 1997. 
[15] M. Buchheit and P. B. Laursen, "High-Intensity interval training, solutions to the programming puzzle. Part 1: cardiopulmonary emphasis," Sports Medicine, vol. 43, no. 5, pp. 313-338, 2013.

[16] American Thoracic Society and American College of Chest Physicians, "ATS/ACCP statement on cardiopulmonary exercise testing," Journal of Respiratory and Critical Care Medicine, vol. 167, no. 2, pp. 211-277, 2003.

[17] M. R. Miller, J. Hankinson, V. Brusasco et al., "ATS/ERS Task force: standardization of lung function testing (2) Standardization of spirometry," European Respiratory Journal, vol. 26, no. 2, pp. 319-338, 2005.

[18] J. Wagner, J. L. Clausen, A. Coates et al., "ATS/ERS Task force: standardization of lung function testing (3) Standardization of the measurement of lung volumes," European Respiratory Journal, vol. 26, no. 3, pp. 511-522, 2005.

[19] N. MacIntyre, R. O. Crapo, G. Viegi et al., "Standardisation of the single-breath determination of carbon monoxide uptake in the lung," European Respiratory Journal, vol. 26, no. 4, pp. 720 735, 2005.

[20] B. J. Noble, G. A. V. Borg, and I. Jacobs, "A category-ratio perceived exertion scale: relationship to blood and muscle lactates and heart rate," Medicine and Science in Sports and Exercise, vol. 15, no. 6, pp. 523-528, 1983.

[21] M. Gayda, E. Normandin, P. Meyer, M. Juneau, M. Haykowsky, and A. Nigam, "Central hemodynamic responses during acute high-intensity interval exercise and moderate continuous exercise in patients with heart failure," Applied Physiology, Nutrition, and Metabolism, vol. 37, no. 6, pp. 1171-1178, 2012.

[22] O. Rognmo, T. Moholdt, H. Bakken et al., "Cardiovascular risk of high- versus moderate-intensity aerobic exercise in coronary heart disease patients," Circulation, vol. 126, pp. 1436-1440, 2012.

[23] D. E. O’Donnell, S. M. Revill, and K. A. Webb, "Dynamic hyperinflation and exercise intolerance in chronic obstructive pulmonary disease," American Journal of Respiratory and Critical Care Medicine, vol. 164, no. 5, pp. 770-777, 2001.

[24] J. A. Neder, P. W. Jones, L. E. Nery, and B. J. Whipp, "Determinants of the exercise endurance capacity in patients with chronic obstructive pulmonary disease: the power-duration relationship," American Journal of Respiratory and Critical Care Medicine, vol. 162, no. 2, pp. 497-504, 2000.

[25] D. E. O’Donnell, C. D’Arsigny, and K. A. Webb, "Effects of hyperoxia on ventilatory limitation during exercise in advanced chronic obstructive pulmonary disease," American Journal of Respiratory and Critical Care Medicine, vol. 163, no. 4, pp. 892898, 2001.

[26] N. D. Eves, L. C. Sandmeyer, E. Y. Wong et al., "Heliumhyperoxia: a novel intervention to improve the benefits of pulmonary rehabilitation for patients with COPD," Chest, vol. 135, no. 3, pp. 609-618, 2009.

[27] I. Vogiatzis, S. Nanas, E. Kastanakis, O. Georgiadou, O. Papazahou, and C. Roussos, "Dynamic hyperinflation and tolerance to interval exercise in patients with advanced COPD," European Respiratory Journal, vol. 24, no. 3, pp. 385-390, 2004. 


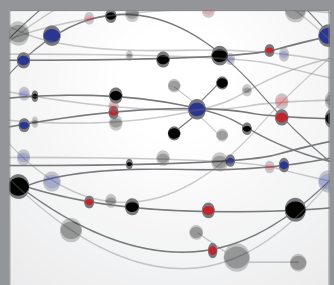

The Scientific World Journal
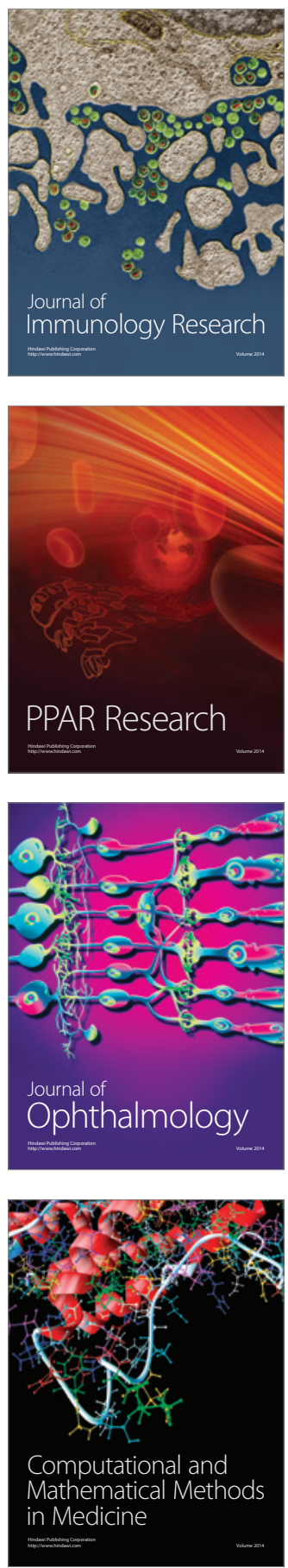

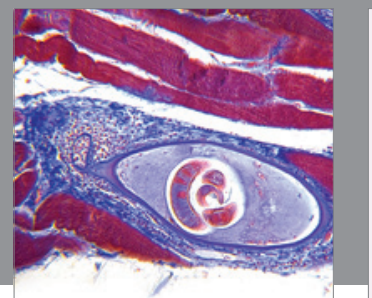

Gastroenterology

Research and Practice
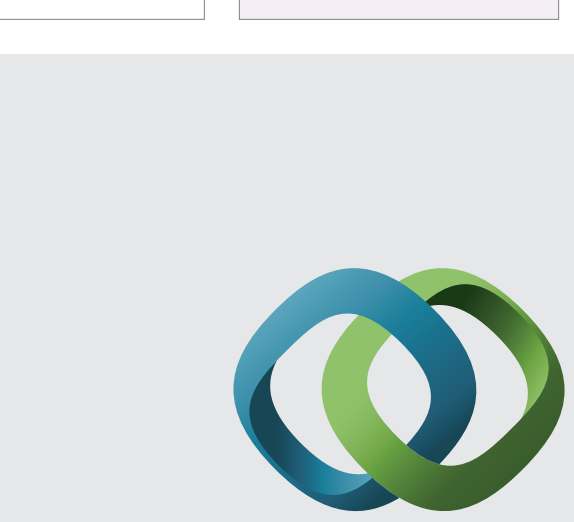

\section{Hindawi}

Submit your manuscripts at

http://www.hindawi.com
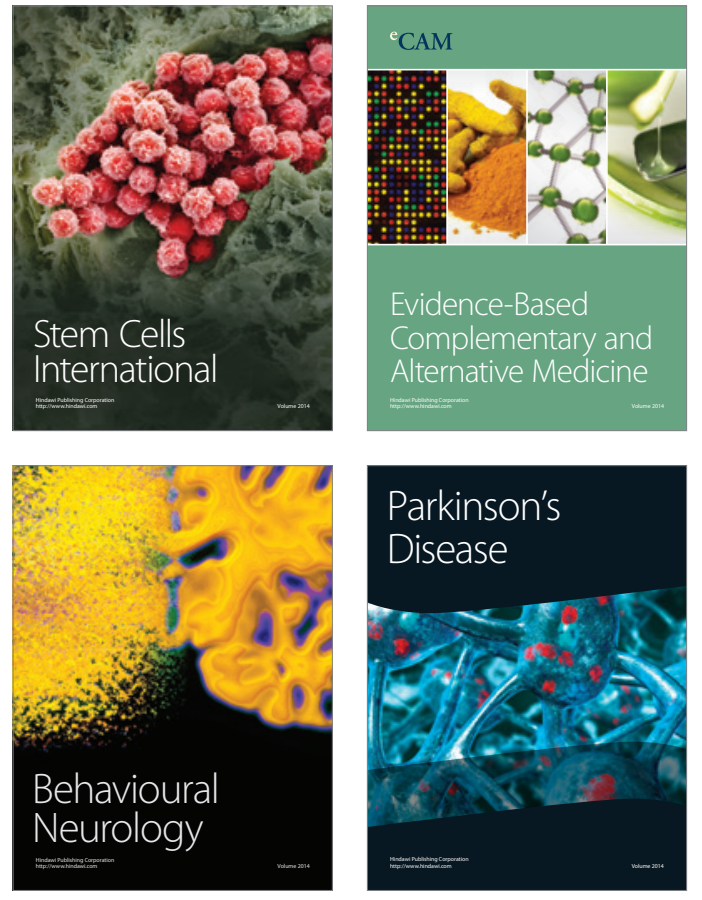
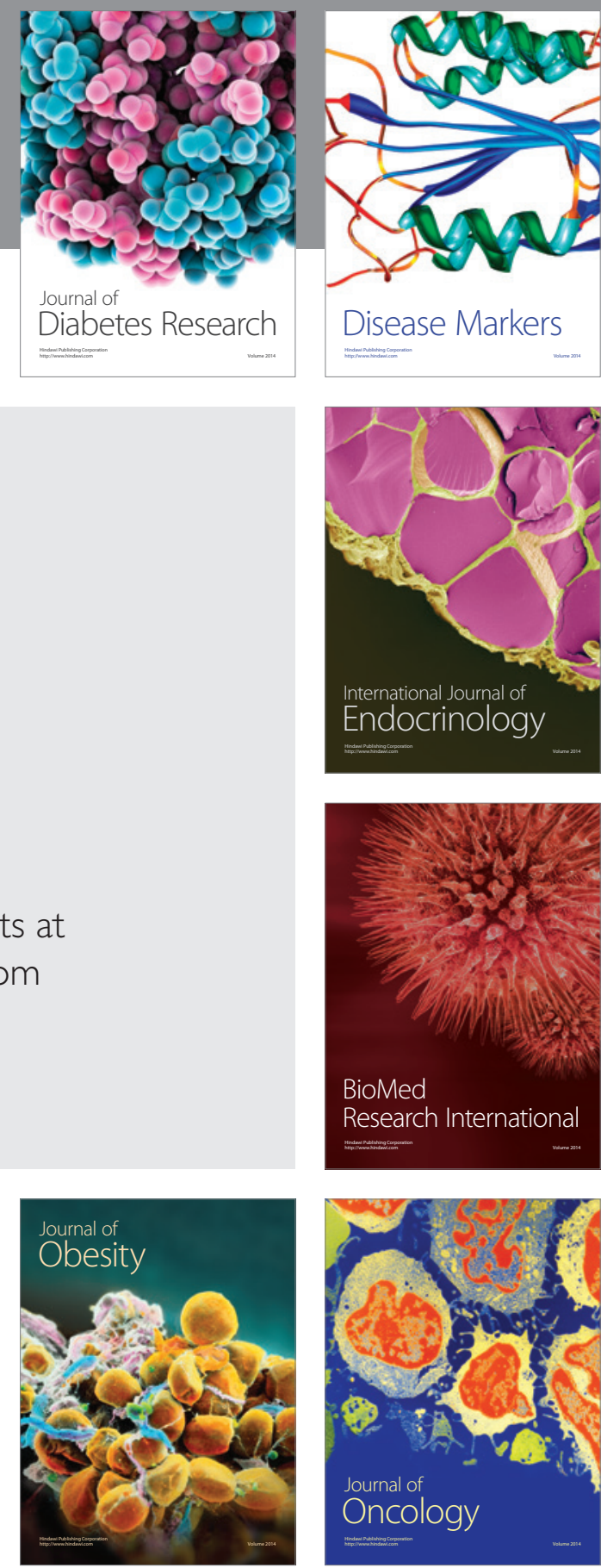

Disease Markers
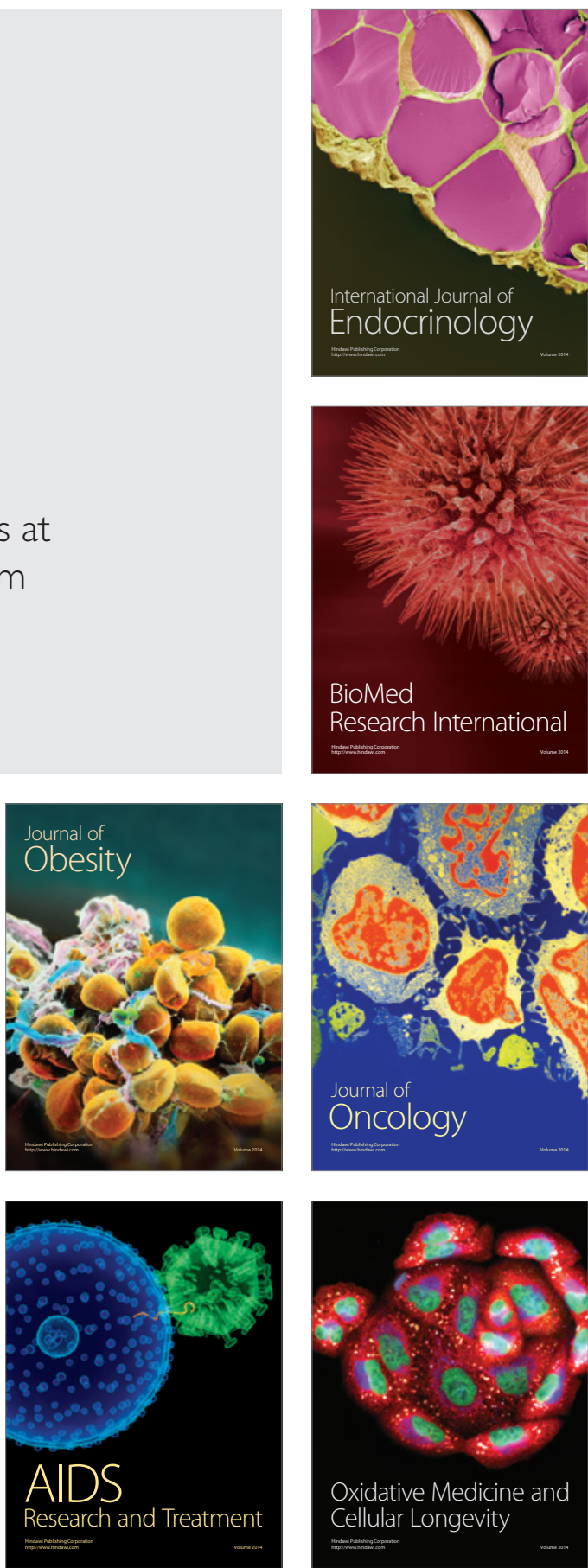